\title{
SPONTANEOUS CAPILLARY PULSATION IN COMPLETE HEART BLOCK
}

BY

\author{
G. A. MACGREGOR*
}

From Hammersmith Hospital and the Department of Medicine, Postgraduate Medical School of London

Received August 4, 1958

Capillary pulsation is a natural phenomenon (Quincke, 1868), often seen in the face and hands of healthy subjects, when the skin is compressed with a glass slide or firmly stroked to produce a flare reaction (Lewis, 1924). It appears especially in hot weather, fevers, and pregnancy, and after exercise, when the skin is warm; and also in aortic regurgitation, and high output states such as anæmia and hyperthyroidism. It is due to transmission of the pulse from the arteries through open capillaries to the subpapillary venous plexus, filling of which largely determines the colour of the skin (Boas, 1924).

An exaggerated form of the sign, which Lewis termed spontaneous capillary pulsation, has been observed in patients with hypertension (Osler, 1892), aortic regurgitation, and arteriovenous fistulæ (Lewis and Drury, 1923). In such cases, rhythmic flushing of skin which has not been stroked or compressed may be visible with each heart beat. According to Lewis, spontaneous pulsation is usually confined to the forehead and cheeks, and is not ordinarily present in normal subjects. Its occurrence in five cases of complete heart block is described here, with further observations made in a series of 50 hospital patients.

\section{CASE RePORTS}

Case 1. A 63-year-old man had had complete heart block for four years when admitted to St. Thomas's Hospital in 1951. His blood pressure was $280 / 90 \mathrm{~mm}$. $\mathrm{Hg}$ and his pulse rate varied between 28 and 60 a minute. With each heart beat, his face and neck flushed so vividly that it was visible from the end of the bed. This effect was most obvious at slow heart rates, and it continued with the regularity of a neon sign, even when all treatment was withheld. It was not therefore due to vasomotor changes induced by the drugs that he had received.

Case 2. A few hours after admission to St. Thomas's Hospital with chest pain in 1953, a man, aged 73, had several syncopal attacks. His pulse rate was 44 a minute and a cardiogram showed complete heart block. His blood pressure was $190 / 70 \mathrm{~mm}$. Spontaneous capillary pulsation was seen on his forehead and cheeks.

Case 3. In 1955, a 64-year-old woman attended Hammersmith Hospital with complete heart block. She had had syncopal attacks for twenty years, and in 1941 her cardiogram showed defective intraventricular conduction. She was pale, but not anæmic, with a pulse rate of 36 a minute and a blood pressure of $240 / 90 \mathrm{~mm}$. Systolic flushing was visible in the pale skin of her forehead, but the sign was more obvious in the palms of her hands. Direct recording of her brachial arterial pulse showed a high pulse pressure, with the upstroke and downstroke less steep than those seen in aortic regurgitation (Fig. 1).

Case 4. An 80-year-old woman had two attacks of complete heart block while in Hammersmith Hospital in 1957. On both occasions spontaneous capillary pulsation appeared. It was seen on her forehead while

\footnotetext{
* Present address: St. Luke's Hospital, Guildford, Surrey.
} 
her pulse rate was 30 a minute and blood pressure $140 / 50 \mathrm{~mm}$. The sign was absent, and it could not be elicited with a glass slide, during sinus rhythm when the pulse rate was 70 to 84 a minute and the blood pressure $120 / 90 \mathrm{~mm}$.

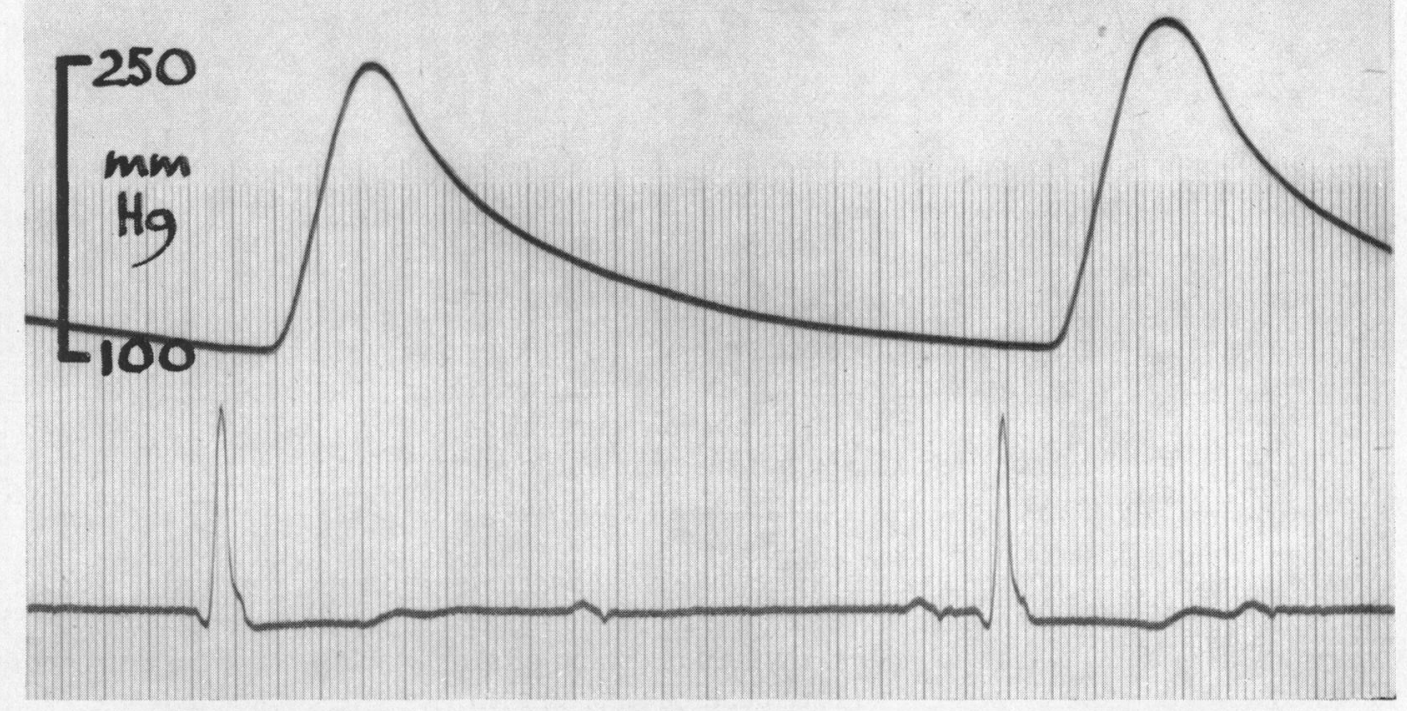

FIG. 1.-A direct recording of the brachial arterial pulse in complete heart block (Case 3). The ventricular rate was 33 a minute.

Case 5. On admission to Hammersmith Hospital in 1957, a 70-year-old woman had a pulse rate of 80 a minute and a blood pressure of $170 / 90 \mathrm{~mm}$. Soon afterwards, she had several Stokes-Adams attacks. Her face paled during asystole, which lasted 5 to 10 seconds, and with the subsequent flush spontaneous capillary pulsation appeared on her forehead. The sign accompanied only the first three or four heart beats, when sinus rhythm (shown by an electrocardiogram) immediately followed ventricular standstill. Asystole was, however, sometimes followed by temporary idioventricular rhythm, and while this continued spontaneous capillary pulsation persisted. In one such attack, the pulse rate was 50 a minute and the blood pressure $195 / 80 \mathrm{~mm}$.

\section{FURTHER OBSERVATIONS}

To obtain an idea of the incidence of spontaneous capillary pulsation, 50 adult patients were examined in July 1957. The unusually hot weather at the time favoured the occurrence of capillary pulsation, and it was detected with the aid of a glass slide in 32 patients, 8 of whom showed the sign spontaneously when the untouched skin of their face and hands was closely inspected in a bright light. Subjective error was eliminated by checking the rate of intermittent flushing with the radial pulse, taken simultaneously by an independent observer. A reason for the prominence of the sign was evident in four patients.

(a) Systolic flushing of the forehead and palms was seen in a woman, aged 54, with aortic regurgitation (P. 88; B.P. 150/50).

(b) A woman, aged 74, with systemic lupus erythematosus who was febrile and anamic, showed systolic flushing of the palms (P. 90; B.P. 190/110).

(c) Flushing appeared in the facial erythema resulting from A.C.T.H. therapy in an asthmatic man, aged 64. (P. 84; B.P. 130/80). 
(d) The sign was also found in an area of recent sunburn erythema on the forehead of a woman, aged 73. (P. 70; B.P. 190/90).

The remaining four patients were also elderly; they had high pulse pressures, but no other reason was found for exaggeration of the sign.

(e) A woman, aged 77, with essential hypertension and atrial fibrillation, showed systolic flushing of the forehead, which was absent with weak pulse beats. (P. 60; B.P. 240/140).

(f) Flushing of the forehead was present in a woman of 69 with ischæmic heart disease. (P. 110; B.P. 210/90).

(g) A healthy woman of 90 showed systolic flushing of symmetrical patches of red skin on her chin. (P. 80; B.P. 220/90).

(h) Systolic flushing of the palms and forehead was seen in a woman, aged 80, admitted with procidentia. (P. 70; B.P. 220/80).

\section{Discussion}

It is not surprising that capillary pulsation is conspicuous in complete heart block, since the functional effects of bradycardia in some ways resemble those of aortic regurgitation. Thus cardiac enlargement, an increased ventricular stroke output, and a wide pulse pressure are common to both conditions. Of greater interest is the fact that the sign appeared in its exaggerated form in five consecutive cases of heart block. Evidently the altered state of the circulation in these patients particularly augmented the "capillary" pulse.

Two circulatory changes that are pronounced in complete heart block are prolongation of diastole and a high pulse pressure. The former effect by itself is not likely to exaggerate capillary pulsation. At a heart rate of 30 a minute, diastole is about three times longer than normal, and emptying of the arterial reservoir is accordingly more complete (Wood, 1956). However, since capillary pulsation ultimately depends upon dilatation of cutaneous arterioles (Lewis, -1927$)$, undue emptying of the arterial system cannot directly accentuate the sign. Nor does the diastolic pause seem long enough in complete heart block for accumulation of katabolites to induce cutaneous vasodilatation. This is the mechanism believed to be largely responsible for the flush which follows Stokes-Adams attacks, but asystole then lasts relatively much longer. To produce visible reactive hyperæmia in the hand, $I$ have found that the circulation must be arrested for at least ten seconds. Prolongation of diastole is certainly not essential in the production of spontaneous capillary pulsation, since the sign appears in patients with rapid sinus rhythm.

The high pulse pressure is a more important factor. Although Lewis (1924) was unable to correlate the appearance of capillary pulsation with the pulse pressure in aortic regurgitation, he noticed that the sign was often most obvious when the pulse pressure was high. Spontaneous capillary pulsation is at least partially dependent upon the pulse pressure, as it failed to accompany weak radial pulse beats in the patient with atrial fibrillation (Case $e$ ). Moreover, the sign disappeared in the two cases of paroxysmal heart block, immediately the pulse pressure diminished with restoration of sinus rhythm. It is also notable that all the control cases showing spontaneous pulsation, except the one with ACTH-induced vasodilatation, had high pulse pressures. The blood pressure elevation in several of them was of the type attributed to increased rigidity, through ageing, of the aorta and great vessels (Wood, 1956). Vascular sclerosis was presumably also present in the cases of heart block, since they were all elderly.

Whether due to an increased volume of blood entering a relatively empty arterial system (as in complete heart block and aortic regurgitation), or to abnormal rigidity of the great vessels, a high pulse pressure thus as a rule accompanies spontaneous capillary pulsation. Rhythmic stimulation of the baroceptors is augmented under these circumstances, and it is conceivable that the resulting increase in reflex vasodilatation accentuates the capillary pulse. 
SUMMARY

Spontaneous capillary pulsation was observed in five consecutive cases of complete heart block, and in 8 out of 50 hospital patients examined during a period of unusually hot weather.

The subjects showing the sign had, with one exception, high pulse pressures. This factor appeared to play an important part in exaggerating capillary pulsation.

I thank Dr. Evan Jones, Dr. C. L. Cope, Dr. C. Fletcher, and Dr. S. Sherlock for permission to report these cases; Prof. J. McMichael for his advice; and Dr. A. Hollman for the arterial pulse tracing.

\section{REFERENCES}

Boas, E. P. (1924). Heart, 11, 57.

Lewis, T. (1924). Heart, 11, 151.

(1927). The Blood Vessels of the Human Skin and their Responses. London.

and Drury, A. N. (1923). Heart, 10, 301.

Osler, W. (1892). Principles and Practice of Medicine. London.

Quincke, H. (1868), quoted by Major, R. H. (1930). Classic Descriptions of Disease. Baltimore.

Wood, P. (1956). Diseases of the Heart and Circulation. London. 